\title{
Polylactic Acid Organogel as Versatile Scaffolding Technique
}

Xavier Punet ${ }^{\mathrm{a}, \mathrm{b}, 1}$,Riccardo Levato ${ }^{\mathrm{a}, \mathrm{b}, 1,2}$, Isabelle Bataille ${ }^{\mathrm{c}, \mathrm{d}}$, Didier Letourneur ${ }^{\mathrm{c}, \mathrm{d}}$, Elisabeth Engel $^{\text {a,b,e }}$, Miguel A. Mateos-Timoneda a,b,e,*

${ }^{a}$ Biomaterials for Regenerative Therapies Group, Institute for Bioengineering of Catalonia (IBEC), Barcelona 08028, Spain.

${ }^{\mathrm{b}}$ CIBER en Bioingeniería, Biomateriales y Nanomedicina (CIBER-BBN), Spain

c INSERM, U 1148, X Bichat Hospital, University Paris Diderot, 46 rue Henri Huchard, 75018 Paris, France

${ }^{d}$ BPC, Institut Galilée, Université Paris 13, 99 avenue JB Clément, 93430 Villetaneuse, France

${ }^{\mathrm{e}}$ Department of Material Science and Metallurgical Engineering, Technical University of Catalonia (UPC), Barcelona 08028, Spain.

KEYWORDS: Poly(lactic) acid, PLA, organogel, nanofibrous, gelation, gel, scaffold.

${ }^{1}$ These authors contributed equally.

${ }^{2}$ Current affiliation: Department of Orthopedics, University Medical Center Utrecht, Utrecht 3584CX, The Netherlands 
ABSTRACT: Tissue engineering requires scaffolding techniques based on non-toxic processes that permits the fabrication of constructs with tailored properties. Here, a two-step methodology based on the gelation and precipitation of the poly(lactic) acid/ethyl lactate organogel system is presented. With this technique nanofibrous matrices that resemble natural extracellular matrix can be easily obtained, while allowing control over the mechanical properties of the device. Gelation temperature and the dynamics of the gelation of the organogel system are characterized, and the final mechanical and viscoelastic properties, as well as porosity, as function of the initial polymer concentration are described. We show that gelation temperature of the system is concentration independent and below $44.5^{\circ} \mathrm{C}$, which permits gelation at room temperature. Furthermore, mechanical properties are found in the range of the soft organic tissues, and the obtained micro-network architecture gives place to a flexible structure. Such structure presents tuneable elastic modulus and viscoelastic properties as function of nanofibers density. Moreover, centimetre-long tubular scaffolds with the diameter of medium-caliber blood vessels were produced. The fibrous nano-architecture mimics the native extracellular matrix fibres diameter and morphology was proven to be suitable to support endothelialization of the lumen of the tube. Thus, this strategy, based on biocompatible green compound might be promising for the fabrication of large 3D scaffolds for tissue engineering applications. 


\section{INTRODUCTION}

Scaffolds play an important role on regenerative medicine owing to their capacity to direct tissue reconstruction by means of physical and chemical cues [1]. However, the scaffolds for tissue engineering on the market are still very limited [2]. Thus, in order to have a real impact on health society, it is very important to develop strategies and products that can be successfully translated toward the clinics. Current challenges for the translation include the development of processes that can control the scaffold physical properties in a tailored way (i.e., architecture, mechanical behaviour, porosity and diffusivity), while, at the same time, can be scaled.

The most used synthetic polymers for scaffold fabrication are the thermoplastic aliphatic polyesters like poly(lactic) acid (PLA), poly(glycolic) acid (PGA) and their co-polymers (PLGA). They have been applied on many biomedical therapies, whether in the form of active scaffold, carriers or holding structures, alone or in combination with other molecules, materials or cells $[3,4]$. Because of their thermoplastic and molecular properties, the techniques explored and used for their processing are numerous [5]. However, most of the known techniques use organic solvents to dissolve the polymer in order to be able to cast, mould or electrospun it, high

temperatures to melt and extrude it. As a consequence, the presence of toxic residues or the degradation of the polymers due to heating, are main drawbacks. For instance, the presence of chloroform in casted scaffolds after one week drying can be up to $5000 \mathrm{ppm}$ [6], while permitted level of residual chloroform for drugs in US is $60 \mathrm{ppm}$ [7]. Another example is the degradation of the molecular chains that PLA suffers during the extrusion or any kind of process that uses heat, which, unless adequately considered during the design of the process, can compromise the final scaffold properties and mechanical behaviour [8,9]. 
Complexity on scaffold manufacturing increases when regulatory requirements are considered. Because cell based biomaterials are strongly regulated and are more costly to implement, acellular biomedical devices are normally preferred, when possible, on industry and clinics [10]. Therefore, such acellular devices have to act as guiding director of the regeneration process. Thus, properties like morphological microstructure and mechanical behaviour need to resemble the natural extracellular matrix of the target tissue, since these physic-chemical properties are known to affect the cell behaviour, growth and differentiation and therefore can be used to promote and direct tissue regeneration [11,12].

Mimicking the extracellular matrix requires structures on the nanometre range, and very few techniques are able to build them. Electrospinning, for instance, allows producing nanosized fibres. However, the obtained structures are usually non-woven and the creation of large, threedimensional constructs, with controlled internal porosity, is limited. To overcome such limitations, is desirable to design alternative processes that can create three-dimensional nanofibrous interconnected porous matrices with good elastic properties and with clinicallyrelevant sizes. This is the case of the organogel phase separation process.

Organogels are defined as gels formed during the immobilization of an apolar phase (usually the solvent) within the spaces of a three-dimensional network formed due to the physical interactions amongst the self-assembled structures of compounds regarded as gelators (the polymer) [13]. These gels are characterized for being thermodynamically stable and having a thermoreversible gelation behaviour which depends on temperature, gelator concentration and gelator molecular structure. Moreover, organogels can present a series of different interesting characteristics like optical and viscoelastic properties, biocompatibility and the capability of being used as drug delivery agents $[13,14]$. 
Among organogels, some are composed by polyester/organic solvent systems. An example of this is the organogel obtained from the entrapment of the tetrahydrofuran (THF) inside a Polylactide (PLA) self-assembled network $[15,16]$. These PLA/THF solution systems can be gelated at low temperatures into a mould. Later, the three-dimensional gel can be phase separated by immersing the gel into water, which displaces the THF. During this exchange, the PLA network from the organogel precipitates due to a phase separation process and a fibrous threedimensional structure is obtained. The organogel phase separation process permits to obtain highly porous scaffolds, with a microstructure that resembles the extracellular matrix, through a very simple and scalable process [16]. However, the use of toxic compounds, such as THF, poses toxicity concerns as well as risk of environmental harming.

The aim of this work was to develop a new organogel system based on the PLA and ethyl lactate (EtLac). EtLac is an FDA-approved compound, which is commonly used in food industry. It is a biodegradable, green solvent, whose degradation products can be metabolized by the human body [17]. For the PLA/EtLac pair, EtLac solvent is known to dissolve the polymer at $40-50{ }^{\circ} \mathrm{C}$, and produce porous structures during its phase separation upon immersion into water [18]. In this work we describe that EtLac is able to allow the formation of a PLA organogel at room temperature, which can be used to produce three-dimensional shapes with control on the nanoarchitecture, porosity and mechanical properties.

This study characterizes a two-step process, based on the PLA/EtLac system, to obtain scaffolds for tissue engineering with high control on their properties and that does not harm the molecular integrity of the material. For such a purpose, the gelation temperature and the gelation dynamics were analysed as a function of polymer concentration. Their correlation with material shrinking upon gelation and, the mechanical and viscoelastic properties of the formed scaffolds, 
as well as their porosity and their diffusion capabilities were analysed. Moreover, a proof-ofconcept of the biological activity of the fabricated scaffolds is presented.

\section{MATERIALS AND METHODS}

\section{MATERIALS}

Poly-L/DL lactic acid 70/30 (Purasorb PLDL 70/38, inherent viscosity midpoint $3.8 \mathrm{dl} / \mathrm{g}$, $\mathrm{Mw} \approx 850,000 \mathrm{Da}$ ) (PLA) was purchased from Purac Biomaterials (The Netherlands).

(L)-Ethyl Lactate (EtLac) 99\% photoresist grade was purchased from Sigma and used without further purification.

\section{POLY(LACTIC) ACID SCAFFOLDS PREPARATION}

Different amounts of PLA were dissolved in EtLac at $54^{\circ} \mathrm{C}$ under stirring. For example, 7 grams of PLA were dissolved in $100 \mathrm{~mL}$ of EtLac to obtain a 7\% PLA solution (w/v). Subsequently, the PLA/EtLac solution was poured into the desired mould and placed at cold temperatures until complete gelation occurred. When the gel was completely formed, it was removed from the mould and immersed into milliQ water. Finally, due to the diffusion of EtLac into water, solvent was exchanged and PLA precipitated by phase separation. The scaffolds were washed several times with water, to remove residual EtLac.

For long-term storage, PLA scaffolds were lyophilized and kept in dry conditions. Before usage, samples were reconstituted in water.

\section{GELATION PROCESS CHARACTERIZATION}

Gelation was characterized by rheology, with a DHR rheometer (TA Instruments) with a Peltier Plate as a temperature control system. 
Viscosity. PLA/EtLac solutions of $4 \%, 7 \%$ and $10 \%$ concentrations were heated until $70{ }^{\circ} \mathrm{C}$ and studied in flow mode. Viscosity was measured for a range of shear rates going from $10^{-5}$ till $10^{3} \mathrm{~s}^{-1}(\mathrm{n}=3)$.

Gel Temperature. The Gel Temperature $\left(\mathrm{T}_{\mathrm{gel}}\right)$ was found following a methodology based on the gelator concentration independency principle [19]. For this purpose, the gelation time as a function of temperature was studied for the different concentrations. Obtained gelation curves were plotted, and the convergence point was found by extrapolation. The gelation times $\left(\mathrm{t}_{\mathrm{gel}}\right)$ needed for the creation of the gelation curves were found by analysing the crossing of the $\tan \delta$ during the gelation process for each sample at each temperature in a tan $\delta$ versus time plot [20]. Studied concentrations ranged from $4 \%$ until $8 \%$ and studied temperatures ranged from $5^{\circ} \mathrm{C}$ until $25^{\circ} \mathrm{C}$. The characterization of the $\tan \delta$ evolution was carried out in oscillatory mode conditions. Frequencies used for the assay were ranged between 0.5 and $2 \mathrm{~Hz}$ and the applied strain was $0.5 \%(n=4)$.

Gelation kinetics. Kinetics was studied in oscillatory conditions, using frequencies ranging between 0.5 and $2 \mathrm{~Hz}$ and a $0.5 \%$ strain. The development and completion of the gelation process was followed by recording the evolution of the storage modulus (G') in time, until a plateau level was reached. PLA/EtLac concentrations of $4 \%, 7 \%$ and $10 \%$ pre-warmed at $50{ }^{\circ} \mathrm{C}$ were used for the analysis, and two different setting temperatures were assayed: $5^{\circ} \mathrm{C}$ and $10^{\circ} \mathrm{C}$. Additionally, $4 \%$ solution pre-warmed at $46^{\circ} \mathrm{C}$ was analyzed as well. For each condition, at least two independent samples were analyzed.

Solution-to-gel shrinkage. The shrinkage occurred during the gelation process was studied through the gap variance regulated by the axial force control of the rheometer. During the gelification process of the sample, the upper plate was set to maintain a constant normal force of 
zero value through the modification of the gap between plates. Therefore, when shrinkage occurred, the sensed force was modified and the gap distance was changed to restore the initial setting of zero value. Thus, the reduction of the gap was recorded in order to measure the volume reduction during the shrinkage. Same settings from kinetics studies were applied $(n=4)$.

\section{PLA SCAFFOLDS CHARACTERIZATION}

Morphology. Material structure was observed by Scanning Electron Microscope (SEM). Images were obtained at intensities between 3-10 kV with a Nova NanoSEM 230microscope (FEI Corporation). Samples were covered with $10 \mathrm{~nm}$ gold by sputtering $(\mathrm{n}=3)$.

Dry weight percentage. Calculated from the ratio between the PLA and the water content. For these purpose, scaffolds were weighted after and before lyophylization $(n=6)$.

Diffusion. Diffusion was analysed by immersing cylindrical scaffolds $(1.2$ diameter x $1.7 \mathrm{~cm}$ height) in an aqueous solutions of rhodamine at $0.05 \%(\mathrm{w} / \mathrm{v})$ concentration. Then, scaffolds were cut transversally in order to observe radial rhodamine internalization. The penetrated distance to the centre was measured by image analysis with ImageJ software. At least two samples per condition were studied, and ten random radial distances per sample were measured. Segments closer to the bases of the cylinder were avoided.

Mechanical assays. Mechanical assays were carried out in a Zwick/Roell BT1-FR0.5TN.D14 testing machine. Dumbbell-shaped samples were fabricated by moulding following the Standard Test Method for Tensile Properties of Plastics (ASTM D 638) and fixed using a 60N Grip system Different PLA/EtLac concentration solutions, ranging between $4 \%$ and $10 \%$, were used to produce test specimens. 
Traction tests were carried out following the ASTM D 638 guidelines at room temperature. From the resulting stress-strain curves, several properties were calculated: Young Modulus (E modulus), tensile strength at yield $\left(\sigma_{\max }\right)$, strain at yield $\left(\varepsilon_{\max }\right)$, work at yield $\left(\mathrm{W}_{\max }\right), 0.2 \%$ offset yield strength ( $\sigma$ at $0.2 \%), 0.2 \%$ offset yield strain ( $\varepsilon$ at $0.2 \%$ ) and $0.2 \%$ offset yield work (W at $0.2 \%)(n=12)$.

Creep-Recovery tests were also carried out. Test specimens were fastened at $1 \%$ of strain at 1\%/s speed. Then, applied stress was maintained constant for 300 seconds and strain $(\varepsilon)$ was recorded. Later, stress was released and maintained at zero constant value. Strain recovery was recorded for an extra 300 seconds. From Creep-Recovery test curves, the following properties were calculated: the Creep Rate ( $\varepsilon / \mathrm{dt})$ during the secondary creep stage (linear creep phase) and the Strain Recovery portion $\left(\mathrm{dL}_{\mathrm{f}} / \mathrm{dL}_{\max }\right)(\mathrm{n}=4)$.

Relaxations test were carried out as follows. Test specimens were fastened at $1 \%$ of strain at $1 \% / \mathrm{s}$ speed. Then, $1 \%$ of deformation was maintained constant for 600 seconds and stress $(\sigma)$ was recorded. Relaxation ratio was calculated between initial and final stress $\left(\sigma_{0} / \sigma_{\mathrm{f}}\right)$. At least four specimens per condition were studied.

\section{CELL CULTURE}

Human umbilical vein endothelial cells (HUVEC) single donor cells (CC-2517, Lonza) were cultured on tubular scaffolds with Supplemented Promocell ${ }^{\circledR}$ SMC Growth Medium 2. Cell adhesion was observed by fluorescence microscopy after $24 \mathrm{~h}$ of culture (initial cell density: 100,000 cells $/ \mathrm{cm}^{2}$ ). Fluorescence labelling was carried out with DAPI (nuclei) and RhodaminePhalloidin (actin cytoskeleton). Cell growth was studied during 13 days of culture (initial cell density: 10,000 cells $/ \mathrm{cm}^{2}$ ). For the observation of cell growth, cultured tubes were embedded 
into paraffin and histological samples were cut along the longitudinal section of the tubes after 1, 5, 9 and 13 day. Cells were stained using Masson's trichrome protocol and observed under light microscopy. For each time point and each condition, at least three independent samples were studied.

\section{STATISTICS}

Experiments were subjected to t-Student testing $(\mathrm{P}<0.05)$. Results were presented as mean \pm standard deviation.

\section{RESULTS}

\section{SCAFFOLD FABRICATION}

PLA/EtLac warmed solution was poured into different moulds, gelated and phase-separated in order to obtain highly porous scaffolds with specific macroscopic shapes. Figure 1 shows a scheme of the fabrication procedure and the macroscopic views of two different scaffolds obtained by such means.

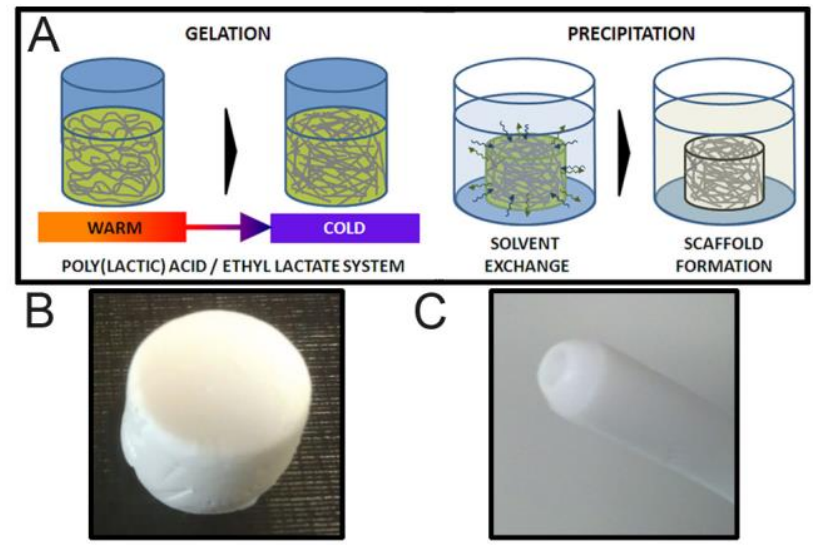

Figure 1. (A) Scheme of the fabrication procedure showing the different steps: gelation and further precipitation to obtain the 3D scaffolds. (B) Lyophilized scaffold obtained using a 24- 
well plate following the described protocol. (C) Hydrated scaffold with a tubular shape used for the in vitro studies.

\section{GELATION PROCESS CHARACTERIZATION}

\section{Viscosity}

For the study of viscosity, temperature was fixed at a working temperature of $70^{\circ} \mathrm{C}$, while shear rate was varied from $10^{-3}$ to $10^{3} \mathrm{~s}^{-1}$ values. Figure 2 showed the viscosity of the different polymer concentrations as a function of shear rate. PLA/EtLac solutions had an inherent viscosity of about 30 to 2000 times the viscosity of water. All solution showed a shear-thinning behavior, until reaching a plateau. It is possible to observe that the higher the polymer concentration is, the lower shear stress is needed to achieve the viscosity plateau.

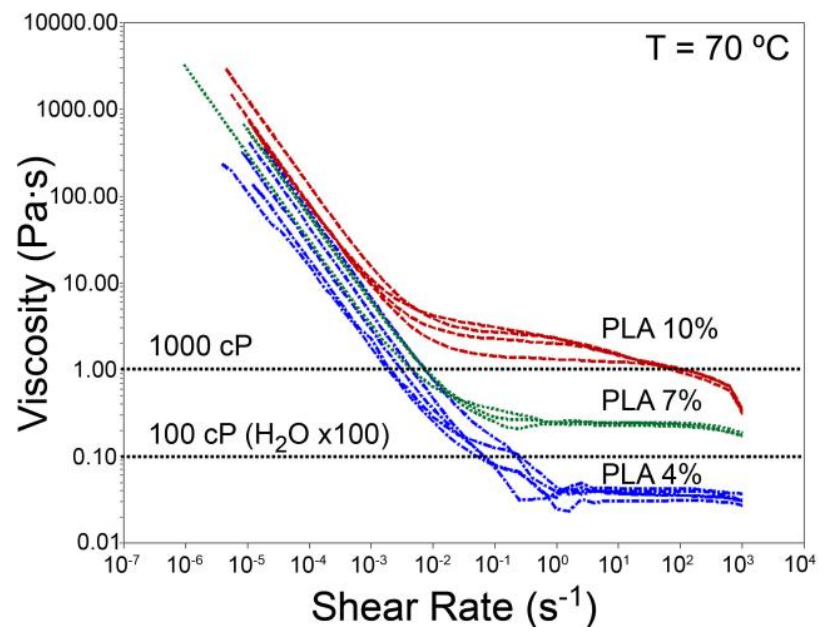

Figure 2. Viscosity plot as function of shear rate for three different PLA/EtLac concentrations $(4 \%, 7 \%$ and $10 \%)$ at $70{ }^{\circ} \mathrm{C}$.

\section{Gel Temperature}

Gelation curves studied by rheometry were plotted, and the concentration independency point was determined (Figure 3). PLA/EtLac concentrations of $4 \%, 6 \%, 7 \%$ and $8 \%$ wereused for the determination of $\mathrm{T}_{\mathrm{GEL}}$. For the crossing point of the different curves, the gel temperature was 
determined at $44.5{ }^{\circ} \mathrm{C}$. This temperature was found by extrapolation following literature procedures $[19,20]$.

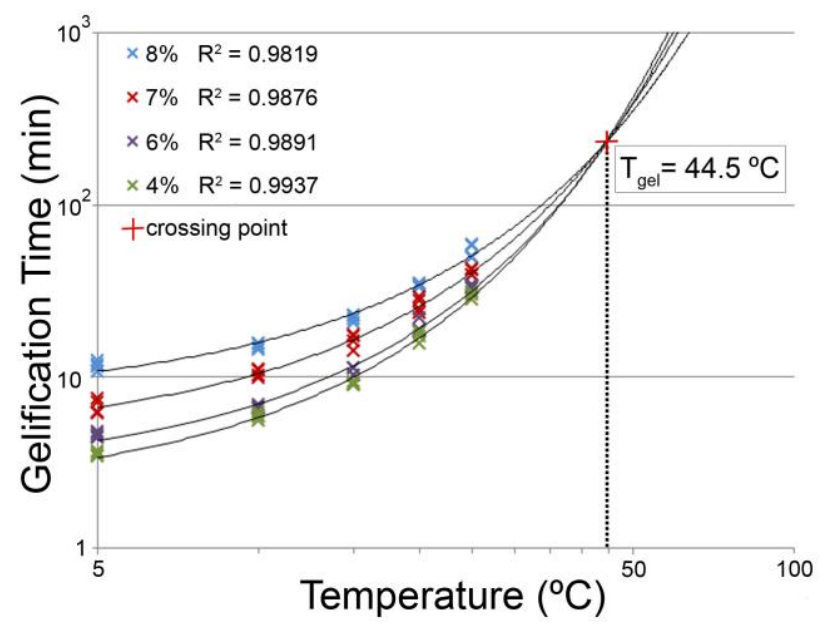

Figure 3. Gelation curves of the PLA/EtLac solutions $(4 \%, 6 \%, 7 \%$ and $8 \% \mathrm{w} / \mathrm{v})$. The extrapolated crossing point defines the $\mathrm{T}_{\mathrm{GEL}}$.

\section{Gelation kinetics}

Parallel to the $\mathrm{T}_{\mathrm{GEL}}$ study, some of the specimens used for the analysis of the gelation time were studied for extended times (after the tan $\delta$ convergence) in order to evaluate the gelation kinetics. On this analysis, the evolution of the storage modulus (G') was recorded and plotted (Figure 4). All gelation curves achieved a plateau level after the completion of the gelation process. This final stable state was dependant on the initial gelator concentration, but independent on the experimental temperature used or the pre-warming applied. In that sense, it was possible to observe that higher concentrations were related to higher levels of storage modulus (higher levels of stiffness). Higher setting temperatures $\left(10^{\circ} \mathrm{C}\right)$ correlated to slower speeds of gelation, this means that more time was needed to achieve the plateau equilibrium. Kinetics also showed, on all samples, a peak on the storage modulus achieved before the plateau 
level. On the first slopes before the peak, it was possible to observe higher slopes (higher speeds of gelation) for higher concentrations. Finally, it was possible to observe for $4 \%$ samples that samples pre-warmed at higher temperatures $\left(50^{\circ} \mathrm{C}\right)$ needed more time to achieve the plateau level than samples pre-warmed at lower temperatures $\left(46^{\circ} \mathrm{C}\right)$.

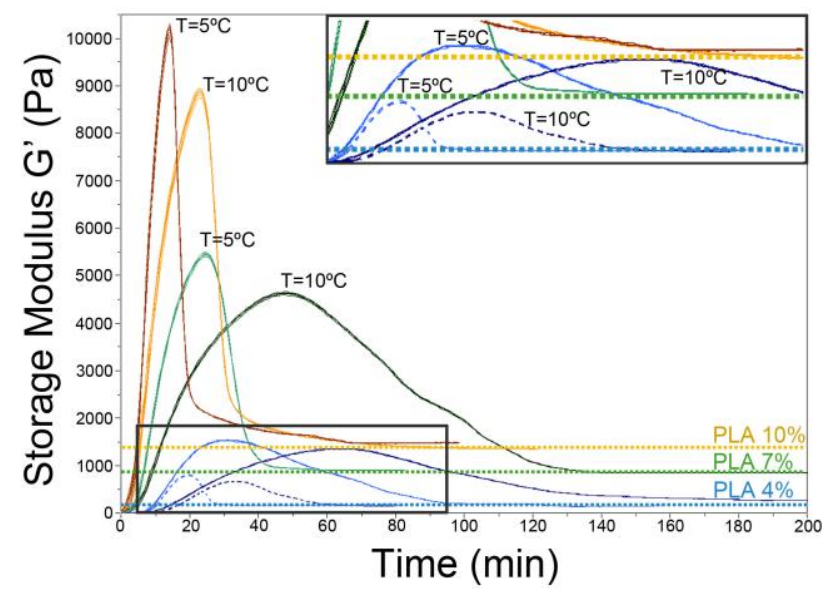

Figure 4. Gelation kinetics curves of the PLA/EtLac 4\% (blue), 7\% (green) and 10\% (brown) solutions. Samples were studied at two different gelating temperatures $\left(5^{\circ} \mathrm{C}\right.$ and $\left.10{ }^{\circ} \mathrm{C}\right)$. Continuous curves stands for samples pre-warmed at $50{ }^{\circ} \mathrm{C}$ and doted curves (only $4 \%$ ) stands for samples pre-warmed at $46{ }^{\circ} \mathrm{C}$.

\section{Solution-to-gel shrinkage}

The shrinkage of the PLA/EtLac solutions during the gelation process is shown in Figure 5. The shrinkage of the gel ranged between $0.8 \%$ and $3.5 \%$, with the larger ratios found in samples with higher polymer concentration.

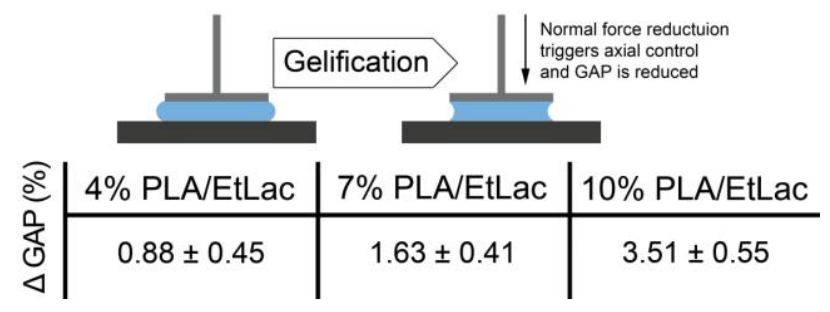


Figure 5. Measured shrinkages presented as gap reduction percentage \pm standard deviation. The cartoon represents how the shrinkage was measure.

\section{PLA SCAFFOLDS CHARACTERIZATION}

The study of gelation kinetics revealed that thermostable gels were obtained at $5^{\circ} \mathrm{C}$ after $2 \mathrm{~h}$ of gelation. Therefore, these settings were selected to obtain gels, which would be used for the formation of scaffolds. These gels, formed from different initial PLA/EtLac concentrations ranging from $4 \%$ to $10 \%$, were used for the creation of solid scaffolds through a two-step protocol. Once the organogels were formed, they were immersed into water in order to exchange the EtLac solvent by water and induce the PLA precipitation through a phase separation process. In this way, scaffolds were obtained, which mechanical, porous and diffusion properties depending on the initial PLA/EtLac concentration.

\section{Morphology}

SEM images from 4\%,7\% and 10\% concentrated samples are shown in Figure 6 . It was not possible to observe clear differences between concentrations, in terms of network density and porosity. All images showed a random structured network conformed by nanofibers ranged below $200 \mathrm{~nm}$. 

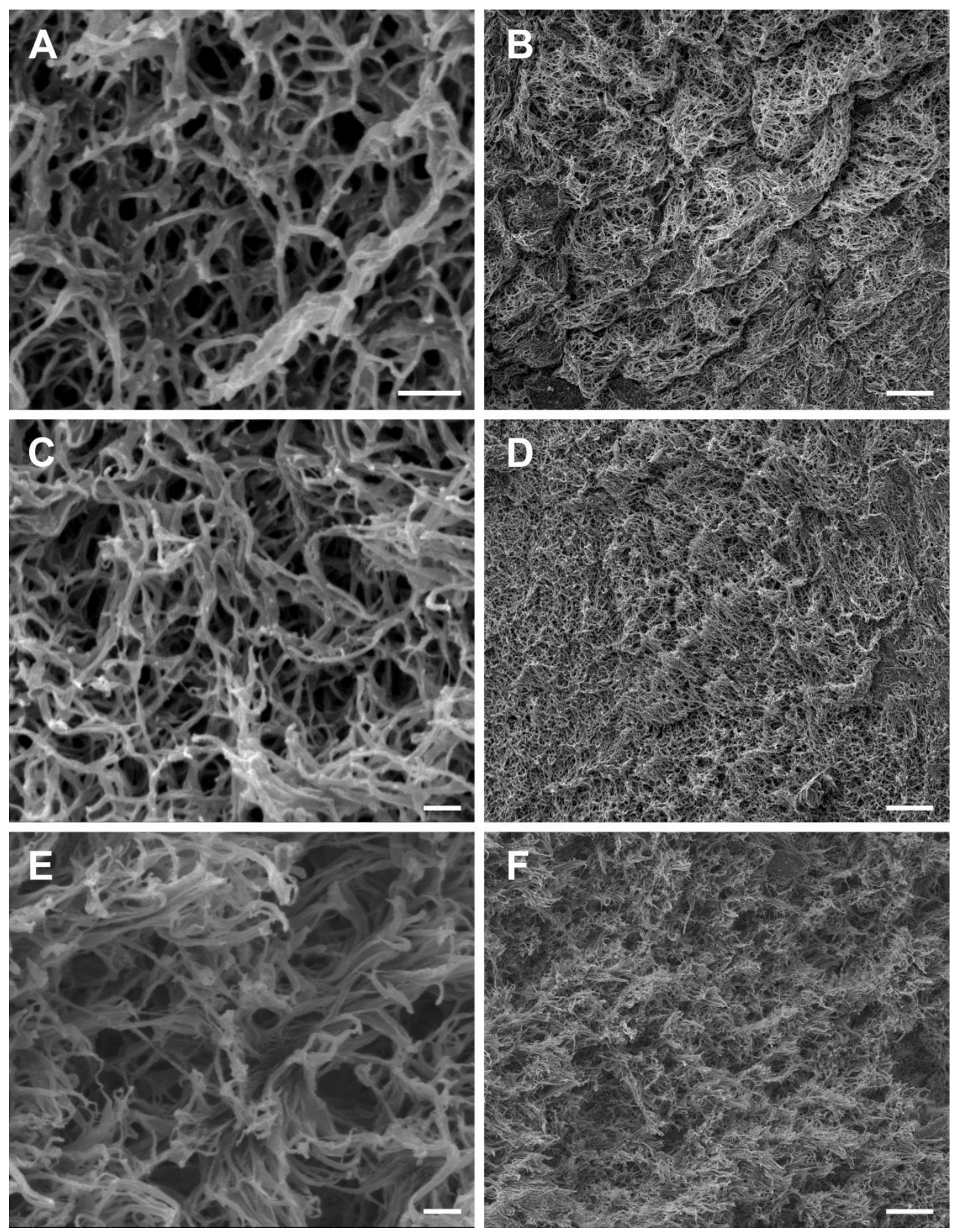

Figure 6. SEM Images of lyophilized scaffolds obtained from PLA/EtLac concentrations of (A-

B) 4\%, (C-D) 7\% and (E-F) 10\%. Scales bars represent $1 \mu \mathrm{m}$ on (A), (C) and (E) images; and 10 $\mu \mathrm{m}$ on (B), (D) and (F) images. 


\section{Dry weight percentage}

In order to study the differences between material densities, the weight of wet scaffolds (with water entrapped inside) and the weight of lyophilized scaffolds were compared. The water entrapped inside represented between the $87.3 \%$ and the $94.6 \%$ of the weight of the scaffold (10\% and 4\% samples respectively) (Table1), which is a clear evidence of the high porosity of the scaffolds and the differences between them.

Table 1. Dry weight percentage of the scaffolds obtained from the different PLA/EtLac ratios.

\begin{tabular}{l|l|l|l}
\hline PLA/EtLac ratio (w/v) & $4 \%$ & $7 \%$ & $10 \%$ \\
Dry weight percentage (\%) & $5.39 \pm 0.04$ & $8.92 \pm 0.03$ & $12.76 \pm 0.06$ \\
\hline
\end{tabular}

\section{Diffusion}

For the diffusion assays, cylindrical scaffolds formed from different concentrations (4\%, 7\% and $10 \%$ ) were immersed into a rhodamine aqueous solution. Scaffolds were taken after $2 \mathrm{~h}, 6 \mathrm{~h}$ and $22 \mathrm{~h}$ and cut transversally (Figure 7). The dye penetration was measured at each time point and the evolution in time was plotted (Table 2). A clear reduction of the dye diffusion was observed, which can be correlated with an increase of the PLA concentration. 


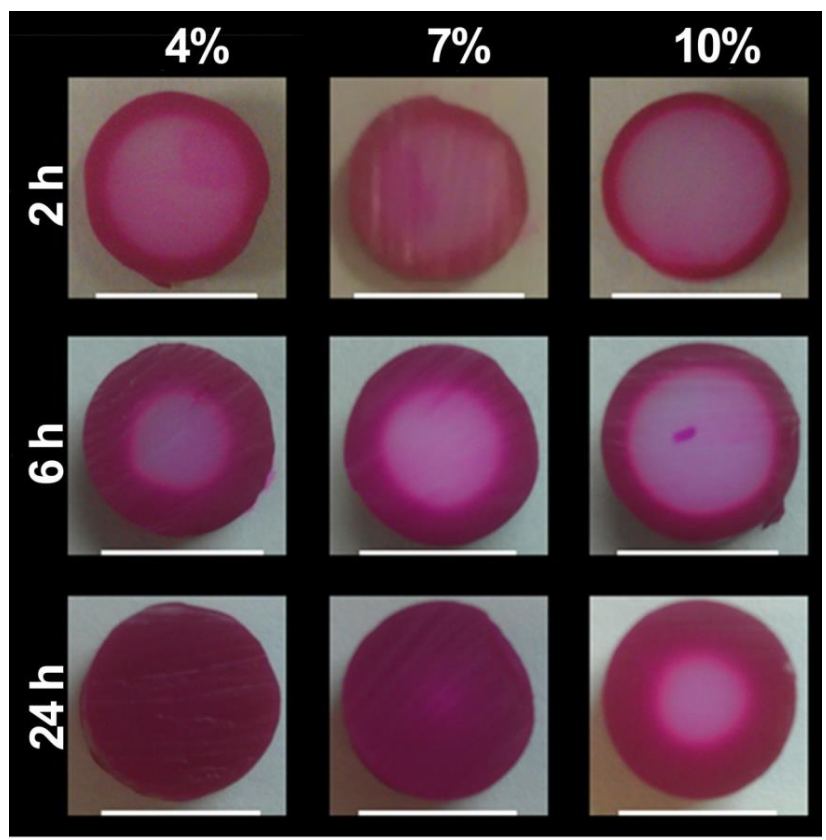

Figure 7. Images of rhodamine diffusion at different time points $(2 \mathrm{~h}, 6 \mathrm{~h}$ and $22 \mathrm{~h})$ for different scaffold compositions (4\%, 7\% and 10\%). Scale bars equal $1 \mathrm{~cm}$.

Table 2. Measured diffusion distance as percentage of the total radius distance $( \pm$ standard deviation).

\begin{tabular}{lccc}
\hline & \multicolumn{3}{c}{ DIFFUSION DiSTANCE (\%) } \\
& $4 \%$ PLA/EtLac & $7 \%$ PLA/EtLac & $10 \%$ PLA/EtLac \\
\hline $2 \mathrm{~h}$ & $22.77 \pm 2.18$ & $22.22 \pm 2.48$ & $14.39 \pm 2.40$ \\
$6 \mathrm{~h}$ & $46.42 \pm 1.46$ & $35.36 \pm 1.92$ & $24.09 \pm 1.39$ \\
$24 \mathrm{~h}$ & $97.64 \pm 3.64$ & $81.06 \pm 3.66$ & $55.41 \pm 1.79$ \\
\hline
\end{tabular}

\section{Mechanical assays}

The mechanical properties of the scaffold were studied. Scaffolds formed from solutions with concentrations framed between the $4 \%$ and the $10 \%$ ratios were tested. 
Traction tests following the ASTM D638 protocol were carried out to evaluate the elastic modulus of the material as a function of the initial PLA/EtLac concentration. The assay was also used to define the tensile properties before rupture, and at $0.2 \%$ yield offset, which is the standard offset that defines the end limit of the elastic region. The elastic modulus increased with the crescent PLA/EtLac percentage. The values of the elastic modulus ranged from 3.69 $\mathrm{MPa}$, to 45.39 $\mathrm{MPa}$ (Figure 8A).

For the tensile properties, it was possible to observe maximum strains ( $\varepsilon$ max) around the $\sim 35 \%$ for all concentrations except the $10 \%$ concentration, which was correlated with a statistically significant reduction of the maximum strain (25.33\%) (Figure $8 \mathrm{~B})$. Instead, strains measured at the $0.2 \%$ yield offset, which represents the elastic limit, were smaller $(2.9 \%$ to $1.35 \%)$ and presented a minimum level after the $7 \%$ concentration (Figure $8 \mathrm{C}$ ). It was also possible to observe that the tensile strength $\left(\sigma_{\max }\right)$ increased from $0.36 \mathrm{MPa}$ to $1.66 \mathrm{MPa}$ for the $4 \%$ and $10 \%$ concentrations respectively (Figure 8D). Instead, strength found at $0.2 \%$ yield offset were four times smaller (0.11 to $0.55 \mathrm{MPa}$ ) (Figure $8 \mathrm{E}$ ). Because stiffness was found to increase along the concentration range, but strain until rupture was not, the energy that the material is able to store showed a maximum at the $8 \%$ concentration $(251.81 \mathrm{~mJ})$ (Figure $8 \mathrm{~F}$ ). However, the energy stored on the elastic deformation range was found to be two orders of magnitude smaller (ranging from 1.34 to $3.23 \mathrm{~mJ}$ ) (Figure $8 \mathrm{G}$ ). 

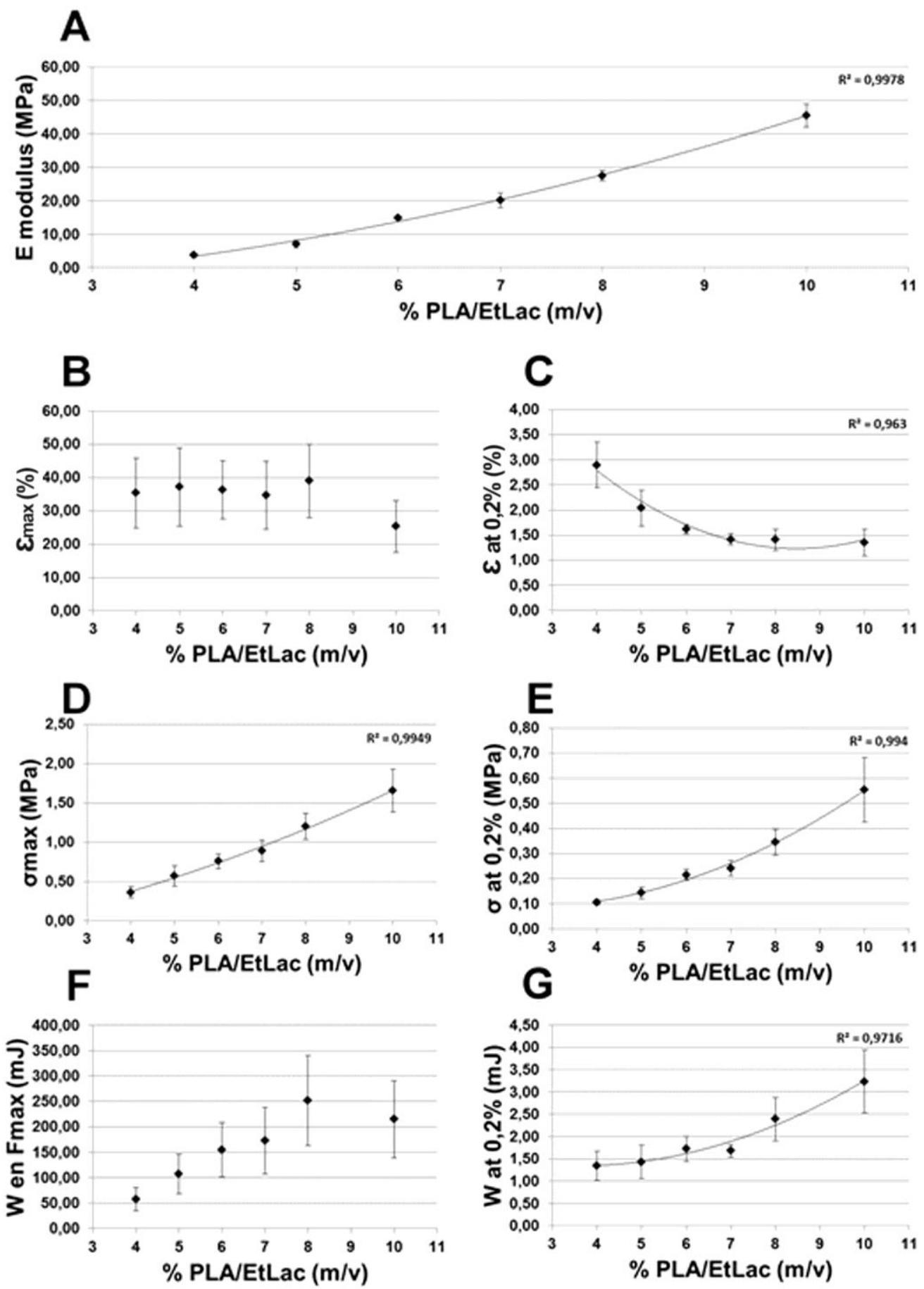

Figure 8. Tensile mechanical properties obtained from scaffolds formed with different PLA/EtLac concentrations. Analyzed properties were (A) Young modulus, (B) strain at tensile strength ( $\varepsilon$ at Fmax), (C) strain at $0.2 \%$ offset ( $\varepsilon$ at $0.2 \%$ ), (D) tensile strength ( $\sigma \mathrm{max})$, (E) stress 
at $0.2 \%$ offset ( $\sigma$ at $0.2 \%),(\mathrm{F})$ Work at tensile strength (W at Fmax) and $(\mathrm{G})$ Work at $0.2 \%$ offset (W at $0.2 \%)$.

Given that the scaffold was composed by a polymer in a fibrous structure with a high level of porosity, it was also necessary to study the viscoelastic behaviour as function of PLA/EtLac ratio. For such a reason, creep and relaxation tests were performed on test specimens with the same dumbbell shape.

The creep test was used to study the speed of deformation that a constant strain produced on test specimens. It is known that materials normally go through three different creep phases. A primary phase in which deformation decelerates, a secondary phase in which deformation stays constant and a tertiary phase in which deformation accelerates and rupture occurs. For this comparison study, the creep rate was measured during the steady phase, this is the secondary phase. Figure 9A shows that specimens obtained from lower concentrated solutions (4\%) presented higher creep rates $(0.2$ strain $\% / h)$ when compared to specimens obtained from higher concentrated solutions (10\%), which presented lower creep rates $(0.03$ strain $\% / h)$. The differences became more significant when it was considered that the stresses used for the creep study, which were obtained by deforming all specimens at $1 \%$ of deformation and setting them constant at this point, resulted to be lower on those samples obtained from lower concentrated solutions (Figure 9B). This means that, even though smaller stresses were applied on $4 \%$ samples, these scaffolds presented higher creep rates compared to $10 \%$ samples.

On the other hand, the strain recovery measured at the end of the creep-recovery tests, showed that $10 \%$ samples allowed up to a $\sim 95 \%$ of strain recovery, while lower concentrated samples like $4 \%$ and $7 \%$ only recovered the $\sim 80 \%$ of the deformation (Figure $9 \mathrm{C}$ ). 

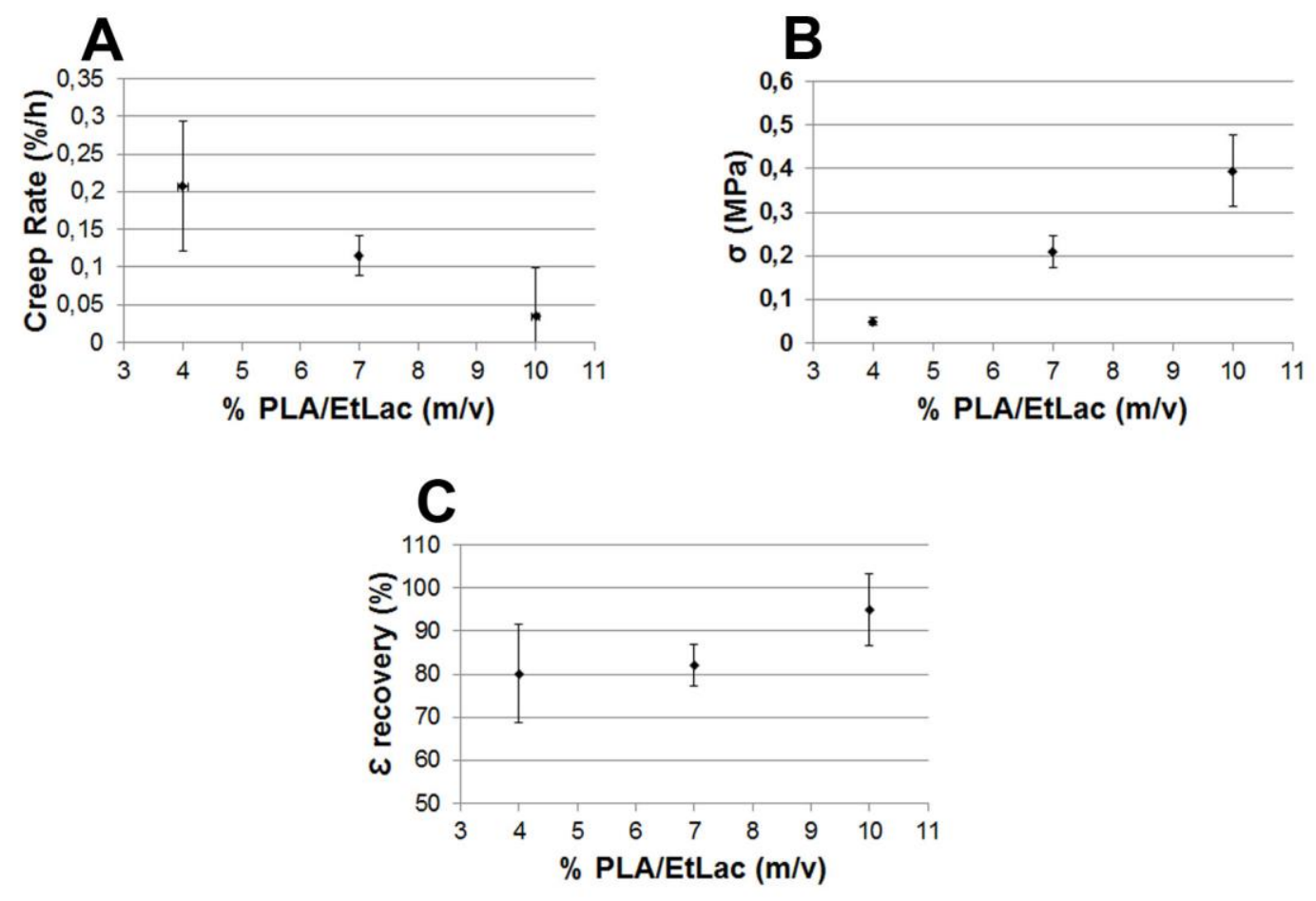

Figure 9. Creep/Recovery test results from different PLA/EtLac concentration. (A) Creep rate evaluated during the secondary creep phase (constant phase). (B) Stresses obtained after 1\% deformation. (C) Recovered strain at the end of the test.

Finally, relaxation tests were carried out to observe the effect of a constant deformation in the rearrangement of the polymeric structure. For this study, samples from different PLA/EtLac ratios were strained and maintained at $1 \%$ deformation. Then, stress relaxation was recorded during 600 seconds and relaxation ratio was measured at the end of test. When results were observed, it was possible to observe that lower concentrations (4\%) correlated with higher relaxation ratios (1.94), and higher concentrations $(10 \%)$ correlated with lower relaxation ratios (1.75) (Figure 10A), which means that viscoelastic effects are more evident for the $4 \%$ samples. These differences became again more significant, when it was considered that for the same 
applied deformation, the $4 \%$ samples showed lower initial stresses than the $10 \%$ samples (Figure 10B).
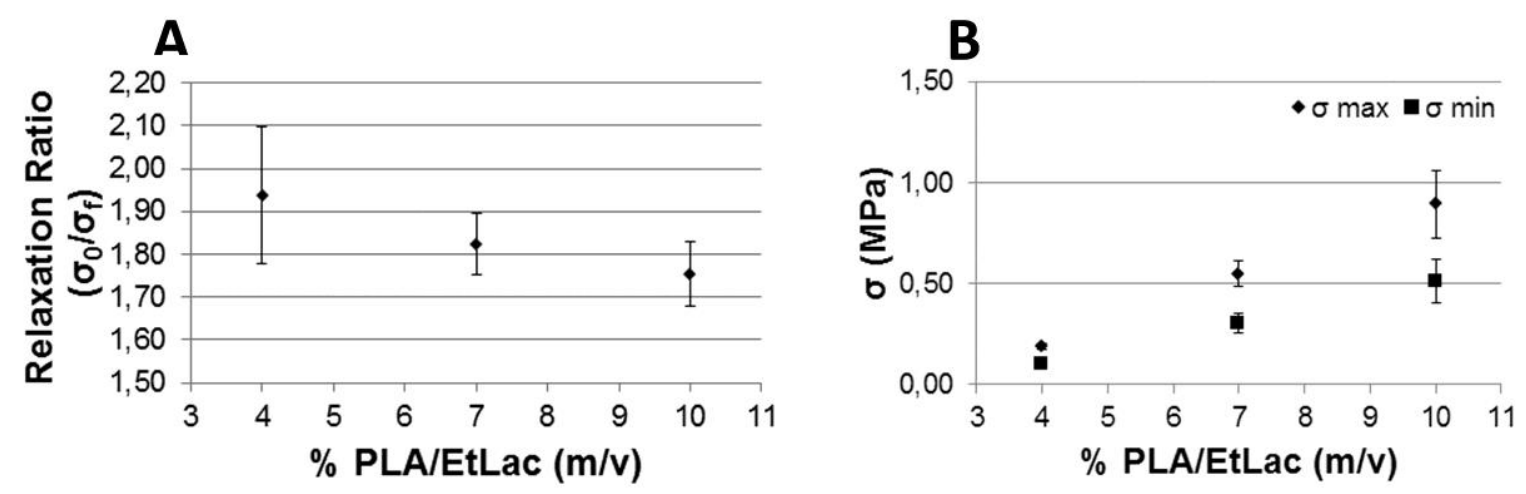

Figure 10. Test relaxation test results from different PLA/EtLac concentration. (A) Relaxation ratio measured from samples fastened at $1 \%$ and maintained for 600 seconds. (B) Stresses obtained at the initial point of the assay.

\section{CELL CULTURE}

Scaffolds with the shape of tubes (internal diameter: $3 \mathrm{~mm}$; external diameter: $4.6 \mathrm{~mm}$ ) were prepared by moulding and then seeded with HUVECs. The study of cell adhesion revealed that cells could firmly attach directly to the scaffold and develop after 24 hours an organized cytoskeleton with the presence of actin stress fibres. In addition, the histology sections of cultured tubes revealed that cells seeded on the scaffolds could survive, proliferate and develop a continuous endothelium layer after 13 days of culture (Figure 11). 

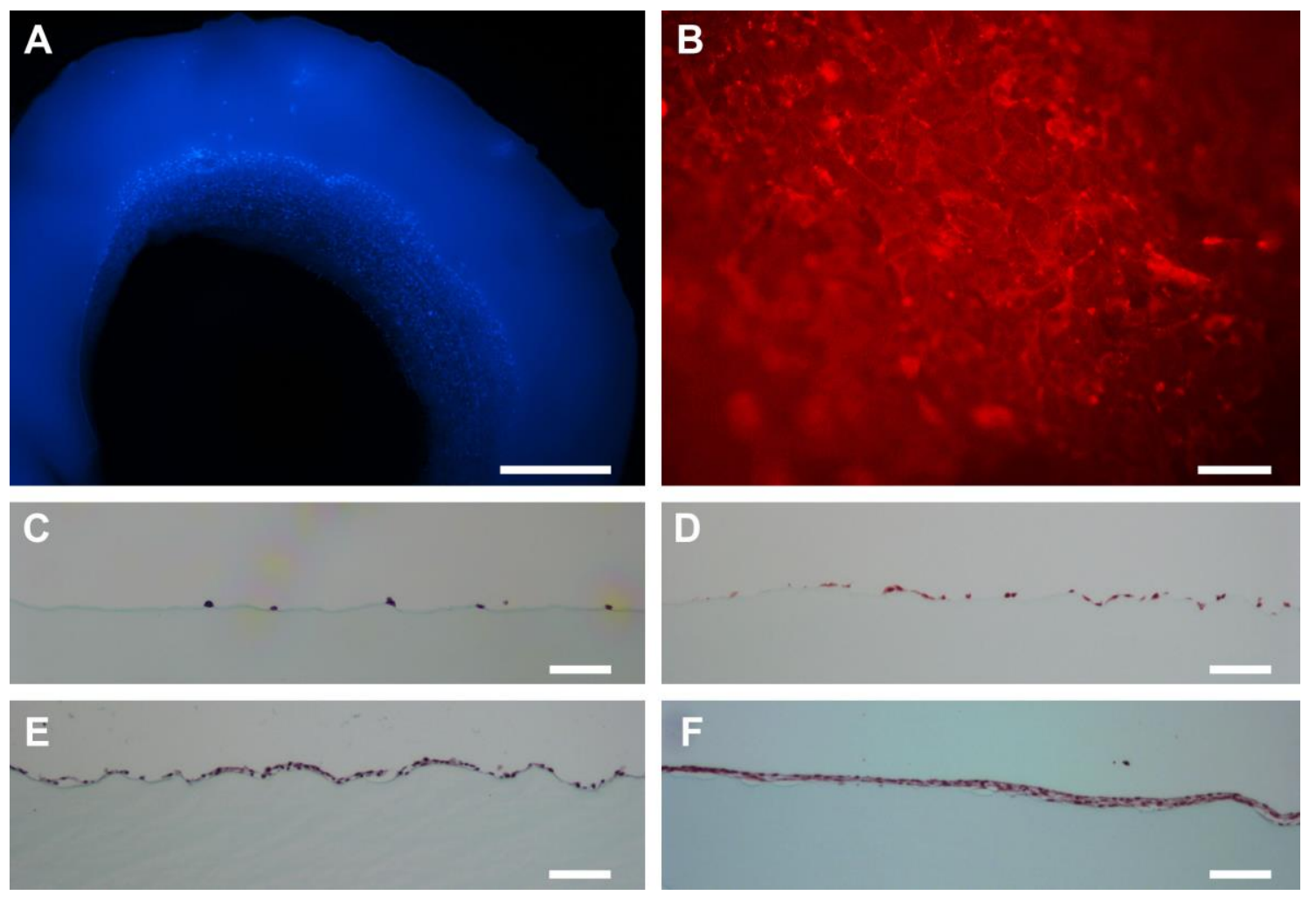

Figure 11. Fluorescence images of stained (A) nuclei and (B) actin cytoskeleton of HUVEC-C cells seeded into the lumen of a tubular scaffold after $24 \mathrm{~h}$ of culture. Histological images of HUVECs cultured into the lumen of tubular scaffolds after 1, 5, 9 and 13 days of culture (C-F respectively). Scale bars stands for $500 \mu \mathrm{m}(\mathrm{A}), 20 \mu \mathrm{m}$ (B) and $100 \mu \mathrm{m}(\mathrm{C}-\mathrm{F})$.

\section{DISCUSSION}

Different parameters affecting the proposed scaffolding technique were studied and characterized in order to gain control on the final properties of the porous material. Viscosity,

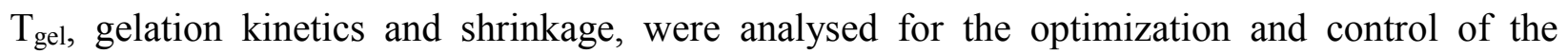
processing, while porosity, diffusion rates, mechanical properties and viscoelastic properties were studied and correlated to the initial polymer concentration. 
Viscosity was studied for its importance and role in many processing methods for fabrication of polymeric devices (i.e. injection and moulding). Thus, the studied range of shear rates was selected because comprised the viscosity behaviour along different states of interest, like the steady state at low shear rates or the stressed state at high shear rates (used for the study of volume flow). Results revealed that liquid PLA/EtLac solutions presented high viscosity levels, from 10 to more than $1000 \mathrm{cP}$. The difference of viscosity between the lower concentration (4\%) and the higher concentration $(10 \%)$ were of about two orders of magnitude. However, for scaffold fabrication process that we propose for our organogels, the most important range is framed between the $10^{-2}-10 \mathrm{~s}^{-1}$ shear rates, which stand for the typical values used on compression and moulding processes. The analysis of the viscosity behaviour revealed that solutions presented a Newtonian region.

Gelation time, instead, depends mainly on polymer concentration and $\mathrm{T}_{\mathrm{GEL}}$. $\mathrm{T}_{\mathrm{GEL}}$, which is an intrinsic value of the PLA/EtLac system, only depends on the molecular structure of the solvent and the gelator, and will be independent of gelator to solvent ratio [19]. Such temperature defines below which level it is possible to obtain a thermostable gel. Thus, when the polymer solution is placed below $\mathrm{T}_{\mathrm{GEL}}$, the gelation process will start at a speed proportional to the difference between $\mathrm{T}_{\mathrm{GEL}}$ and selected temperature. Above $\mathrm{T}_{\mathrm{GEL}}$, instead, the system is a liquid polymer solution, which can be easily mixed, injected or moulded.

Since the gelation is temperature dependent, it is complicated to find the $\mathrm{T}_{\mathrm{GEL}}$ by direct observation. The reason is that gelation speeds near the vicinity of $\mathrm{T}_{\mathrm{GEL}}$ occur at very low rates. Therefore, indirect methodologies have to be used. There are several techniques to analyse the $\mathrm{T}_{\mathrm{GEL}}$, but most of them strongly underestimated it $[19,21]$. One proved strategy for the accurate determination of $\mathrm{T}_{\mathrm{GEL}}$ is based on the extrapolation of gelation curves in a time-temperature 
graph, and the determination of the convergence point of different gelator concentration curves. This crossing point, which defines the gel temperature, is a temperature point in which polymer to polymer interactions are breaking and reforming at the same rate (gelation point), and therefore, the concentration of polymer is inconsequential [19]. Thus, the concentration independency of such a temperature point reveales the $\mathrm{T}_{\mathrm{GEL}}$.

In order to create the gelation time versus temperature graph as a function of concentration, there was a need to find a technique that precisely determines when the gelation of a specific solution for a specific temperature occurred. The study of the crossover between the loss modulus (the viscous component, G') and the storage modulus (the elastic component, G') is commonly used to determine the point in which a solution started to behave like a solid, this is, started to gelate. However, many studies have pointed out that the G'-G' crossover point correlated with the precise gelation point only for a few number of cases [22]. In order to avoid such mismatch, we studied the evolution of $\tan \delta\left(G^{\prime \prime} / G^{\prime}\right)$ along time in a multifrequency rheology test, which is a more accurate strategy to determine the $\mathrm{T}_{\mathrm{GEL}}$ [20]. It was thus possible to corroborate the concentration independency of the $\mathrm{T}_{\mathrm{GEL}}$ point and determine its value at 44.5 ${ }^{\circ} \mathrm{C}$. The accurate definition of the $\mathrm{T}_{\mathrm{GEL}}$ value was relevant not only for the fixation of the minimum working temperature and maximum gelation temperature, but also for the optimization of the gelation process. This process is thermodynamically guided and molecules rearrange and pack to achieve a lower energetic state. Therefore the pre-warming of a PLA/EtLac solution can modify the initial energetic state of the system, and affect the consequent gelation step. This means that, for instance, a solution pre-warmed at $60^{\circ} \mathrm{C}$ will acquire a higher energetic state than a solution pre-warmed at $50^{\circ} \mathrm{C}$, and in turn will need more time to dissipate the energy and achieve the thermostable gel state when this is cooled down. 
Regarding the gelation kinetics, it was interesting to notice that temperature used for the gelation process determines the time needed for the gelation to finish, but had no effect on the final equilibrium state. This information reveals that, for the formation of gels, any temperature below $\mathrm{T}_{\mathrm{GEL}}$ (like room temperature) can be used without changing the final gel properties. This is an interesting property, because, compared to the PLA/THF system [15], the PLA/EtLac system can perform at different temperatures above de $0^{\circ} \mathrm{C}$. Instead, PLA/THF system had to adjust gel setting temperature around $-18^{\circ} \mathrm{C}$ in order to achieve nanofibrous structure, while gelating temperatures near room temperature gave place to platelet-like structures with notably lower mechanical properties [15].

Finally, the presence of different Storage Modulus states achieved before the final equilibrium state (the observed peaks) remained opened to further studies. However, it is interesting to point that the studied gels achieved higher levels of elastic moduli (up to five fold) before the final plateau. Moreover, the evolution of the delta angle ( $\delta$ ) (diphase between stress and deformation) along the gelation process (not shown) indicated that, during the non-equilibrium states found before the plateau, samples already acted like an almost pure Hookenian solid $\left(\delta^{\circ} \approx 0\right)$. This means that, before achieving the plateau, gels were already formed but in a non-equilibrium state. Therefore, these results pointed to the possibility of obtaining stiffer gels (and potentially, stiffer scaffolds) by stopping the gelation process at some time point before the equilibrium state (like the maximum peak), through the precipitation of PLA in water.

Because the gelation of the PLA/EtLac solution follows the reorganization and interaction of the polymeric chains between themselves, it was expected to observe volume shrinkage during the gel formation. Higher polymer concentrations were correlated with higher volume shrinkages, as observed during the rheological analysis of the system. These shrinkage, which 
ranged from $0.8 \%$ to $3.5 \%$, were small but of relevance in designing terms. For instance, for the formation of the dumbbell specimens, the thickness variation due to the shrinkages had to be considered in order to maintain the requirements defined on the ASTM guidelines.

It was expected to correlate the distinct levels of G' with different microstructure and degree of porosity of the final device after precipitation of the PLA in water. However, via the observation of SEM images it was not possible to appreciate differences in porosity. In alternative, the ratio between the solid phase (fibrous PLA structure) and the liquid phase (entrapped water), was measured. We confirmed the higher density of the solid phase on $10 \%$ samples and the high porosity obtained. For the lower concentrations, up to $95 \%$ of the weight was water. Such a value is of high relevance because have strong consequences on the amount of material needed for the obtaining of scaffolds and the amount of water entrapped inside, as well as strong implications on the final specific surface area. For instance, for the PLA/THF system, the surface-to-volume ratio obtained with this nanofibrous networks were up to two orders of magnitude higher than other techniques [15]. Morphologically, all samples displayed a random nanofibrous structure with a submicron-scale porosity suitable to allow the diffusion of metabolites, while restrict cell penetration. This characteristic could be useful on applications in which cells have to be compartmentalised, while other applications in which the scaffold have to be colonized by cells, would require the use of porogen agents to form larger cavities or the implementation of the technique into a solid free-form processing. The obtained continuous nanofibrous network, observed on SEM images, is an architecture very difficult to replicate by means of other techniques in the nanometer size. Moreover, the structure and size of the obtained fibres closely resemble that of natural collagen fibrils $(50-200 \mathrm{~nm})$ [23]. Furthermore, nanofibrous matrices are 
a key factor to promote specific protein adsorption from serum, as well as to control cell attachment, proliferation, development and migration [24].

To further characterize the porous, nanofibrous network, we performed diffusion assays, which were able to detect differences between specimens, with higher PLA concentrations showing reduced the diffusion rate compared to more permeable scaffolds prepared with solution with lower PLA concentration. These results suggest the possibility to use the produced matrices also as devices for drug encapsulation and controlled release, as well as the potential to tune masstransport properties across the device itself.

Mechanical analysis showed that the obtained porous structure allowed the creation of flexible PLA based scaffolds. These scaffolds presented lower elastic modulus and higher deformations than common non-porous PLA, whose tensile modulus are normally found around the $3 \mathrm{GPa}$ and deformations at break around 4\% [25]. Instead, the elastic modulus of the obtained scaffolds could be tuned in the range of 5-50 MPa with maximum deformations around 35\%, which made this material adequate to be used for the regeneration of soft tissues. Indeed, as it has been observed on previous studies [26], the nanofibrous network structure permits to delocalize the applied stresses on the fibrous network in such a way that forces can be shared and dissipated in part throughout the network before the ultimate failure of the material. Such delocalization permits a more flexible deformation and avoids rigidness and brittleness. Thus, with the technique reported in this study, it is possible to obtain PLA porous scaffolds with higher deformations ranges than the ones obtained by means of other architectures, such as electrospun [27] or foamed scaffolds [28-30].

Despite the good mechanical properties shown before rupture, it is important to notice that the properties at the $0.2 \%$ yield offset were a limiting factor. The stresses, deformations and energies 
that the material was able to support before entering the plastic deformation range were remarkably lower than the ones that could support at the fracture limit. For instance, it is important to point that beyond deformations of about 1-3\% (depending on the concentration used for the scaffold formation), the material entered into the plastic regime. Therefore, these short elastic ranges did not make suitable this material for applications that needed larger ranges of elastic deformation, such as skin (10-40\%) and mesentery membrane ( $100 \%$ deformation) [31]. It should be pointed out that could be possible to work with PLA concentrations higher than $10 \%$, to further achieve a wider elastic range and a higher tensile modulus. Moreover, mixtures of smaller and higher molecular weight of PLA could be also used in order to obtain a wider degree of tunability on the mechanical, viscoelastic, porous, diffusion and microstructure properties. However, it should be taken into account, that the addition or change of the molecular size of the gelator would change as well the $\mathrm{T}_{\mathrm{GEL}}$ and the gelation dynamics.

Another interesting property given by this nanofibrous network was the possibility of tuning and modifying the viscoelastic properties. Because the final obtained scaffold was constituted by a polymeric material, viscoelastic effects were expected. However, depending on the network density, the applied stresses and deformations were distributed through different cross-sectional areas, and the water content also contributes to poroelastic properties. As a consequence, specimens of lower concentration presented higher creep rates and relaxation ratios, opposed to specimens of higher concentrations.

Interestingly, viscoelastic properties were able to be adjusted by only modifying the polymer concentration. Such an easy malleability showed potential uses for the designing of materials which are able to mimic the mechanical and viscoelastic properties of different tissues. This is particularly relevant for tissue engineering, since it has been proven that viscoelastic properties 
are also subject to cell mechanosensing and can regulate cell proliferation and differentiation [32].

Finally, the proof-of-concept cell studies on tubular scaffolds evidenced that the produced matrices offer a permissive environment for cell attachment and proliferation. In this case, HUVEC endothelial cells were seeded on the lumen of a PLA tubular scaffold obtained from the precipitation of a $6 \%$ PLA/EtLac organogel system. Fluorescence images showed that cells can firmly attach on the fibrous PLA network, and histological images showed that cells could grow and develop into a continuous cellular endothelium, which covered the entire lumen in a compartmentalized manner. This assay proved the versatility of the system to produce scaffolds by moulding and the correct development of cellular cultures seeded on them along time.

\section{CONCLUSIONS}

This study presents a strategy to generate nanofibrous, matrices of virtually any shape and size, starting from PLA organogels, in a green, non-harmful solvent. An extensive characterization of the scaffold formation process has been provided. Viscosity as a function of shear rate has shown that PLA/EtLac solutions behave like a Newtonian liquid in a range of stresses typical from moulding, pouring, mixing and pumping processes. The determination of the gelation kinetics and $\mathrm{T}_{\mathrm{GEL}}$ has allowed the optimization of the processing temperature for moulding and subsequent gelation, the latter performable at room temperature. Mechanical, structural and diffusivity properties of the obtained solid scaffolds can be tuned simply varying the initial gelator/solvent (PLA/EtLac) ratio. The different gelators to solvent ratios were correlated to different structural network densities, even though all the studied concentrations were able to produce scaffolds with a nanofibrous and highly porous structure. Such peculiar nanoarchitecture 
conferred to the PLA scaffolds a high flexibility and tuneable elastic modulus, as well as the possibility to achieve different viscoelastic responses.

Finally, the methodology presented here, based on the use of green, biocompatible, biodegradable and resorbable materials has proved to be a flexible technology to produce scaffolds for tissue engineering.

\section{AUTHOR INFORMATION}

\section{Corresponding Author}

* E-mail: mamateos@ibecbarcelona.eu.

\section{ACKNOWLEDGMENTS}

The authors acknowledge the financial support of the Spanish Ministry of Economy and Competitiveness (MINECO) through the grants MAT2012-38793 and MAT2015-68906-R.

\section{ABBREVIATIONS}

CCR2, CC chemokine receptor 2; CCL2, CC chemokine ligand 2; CCR5, CC chemokine receptor 5; TLC, thin layer chromatography.

\section{REFERENCES}

[1] F. Edalat, I. Sheu, S. Manoucheri, A. Khademhosseini, Material strategies for creating artificial cell-instructive niches, Curr. Opin. Biotechnol. 23 (5) (2012) 820-825.

[2] S.J. Hollister, Scaffold Design and Manufacturing: From Concept to Clinic, Adv. Mater. 21 (32-33) (2009) 3330-3342. 
[3] L.S. Nair, C.T. Laurencin, Biodegradable polymers as biomaterials, Prog. Polym. Sci. 32 (8-9) (2007) 762-798.

[4] H. Ueda, Y. Tabata, Polyhydroxyalkanonate derivatives in current clinical applications and trials, Adv. Drug. Deliv. Rev. 55 (4) (2003) 501-518.

[5] D.W. Hutmacher, Scaffolds in tissue engineering bone and cartilage, Biomaterials 21 (24) (2000) 2529-2543.

[6] R.A. Giordano, B.M. Wu, S.W. Borland, L.G. Cima, E.M. Sachs, M.J. Cima, Mechanical properties of dense polylactic acid structures fabricated by three dimensional printing, J. Biomat. Sci.-Polym. E8 (1996) 63-75.

[7] Federal Register. Q3C Impurities: Residual Solvents. US Department of Health and Human Services: Food and Drug Administration, Federal Register 62 (1997) 67377-67388.

[8] Y. Wang, S.D. Pacetti, C.V. Harold, Bioabsorbable polymeric stent with improved structural and molecular weight integrity, Patent WO2009155206 A3, 2009.

[9] F.D. Kopinke, M. Remmler, K. Mackenzie, M. Moder, O. Wachsen, Thermal decomposition of biodegradable polyesters - II. Poly(lactic acid), Polym. Degrad. Stabil. 53 (3) (1996) 329-342.

[10] J.P. Pirnay,; A. Vanderkelen, D. De Vos, J.P. Draye, T. Rose, C. Ceulemans, N. Ectors, I. Huys, S. Jennes, G. Verbeken, Business oriented EU human cell and tissue product legislation will adversely impact Member States' health care systems, Cell Tissue Bank 14 (4) (2013) 525560. 
[11] E.S. Place, N.D. Evans, M.M. Stevens, Complexity in biomaterials for tissue engineering, Nat. Mater. 8 (6) (2009) 457-470.

[12] F. Edalat, I. Sheu, S. Manoucheri, A. Khademhosseini, Material strategies for creating artificial cell-instructive niches, Curr. Opin. Biotechnol. 23 (5) (2012) 820-825.

[13] S. Sahoo, N. Kumar, C. Bhattacharya, S.S. Sagiri, K. Jain, K. Pal, S.S. Ray, B. Nayak, Organogels: Properties and Applications in drug delivery, Des. Monomers Polym. 14 (2) (2011) $95-108$.

[14] S. Murdan, Organogels in drug delivery, Expert. Opin. Drug. Deliv. 2 (3) (2005) 489-505.

[15] P.X. Ma, R. Zhang, Synthetic nano-scale fibrous extracellular matrix, J. Biomed. Mater. Res. 46 (1) (1996) 60-72.

[16] J. Hu, X. Sun, H. Ma, C. Xie, Y.E. Chen, P.X. Ma, Porous nanofibrous PLLA scaffolds for vascular tissue engineering, Biomaterials 31 (31) (2010) 7971-7977.

[17] F. Mottu, A. Laurent, D.A. Rufenacht, E. Doelker, Organic solvents for pharmaceutical parenterals and embolic liquids: a review of toxicity data, PDA J. Pharm. Sci. Technol. 54 (6) (2000) 456-469.

[18] R. Levato, M.A. Mateos-Timoneda, J.A. Planell, Preparation of biodegradable polylactide microparticles via a biocompatible procedure, Macromol. Biosci. 12 (4) (2012) 557-566.

[19] S.M. Tosh, A.G. Marangoni, Determination of the maximum gelation temperature in gelatin gels, Appl. Phys. Lett. 84 (21) (2004) 4242-4244. 
[20] J. De Vicente, Polymer Gel Rheology and Adhesion, in: A.M. Grillet, N.B. Wyatt, L.M. Gloe, Rheology, InTech: Croatia, 2012, pp. 59-80.

[21] S.Y. Kim, D.G. Choi, S.M. Yang, Rheological Analysis of the Gelation Behavior of Tetraethylorthosilane/Vinyltriethoxysilane Hybrid Solutions, Korean J. Chem. Eng. 19 (1) (2002) 190-196.

[22] F. Chambon, H.H. Winter, Linear Viscoelasticity at the Gel Point of a Crosslinking PDMS with Imbalanced Stoichiometry, J. Rheol. 31 (1987) 683.

[23] J. Lee, M.J. Cuddihy, N.A. Kotov, Three-dimensional cell culture matrices: state of the art, Tissue. Eng. Part B Rev. 14 (1) (2008) 61-86.

[24] K.M. Woo, V. J. Chen, P.X. Ma, Nano-fibrous scaffolding architecture selectively enhances protein adsorption contributing to cell attachment, J. Biomed. Mater. Res. A 67 (2) (2003) 531-537.

[25] S. Farah, D.G. Anderson, R. Langer, Physical and mechanical properties of PLA, and their functions in widespread applications - A comprehensive review, Adv. Drug. Deliv. Rev. 107 (2016) 367-392.

[26] U. Stachewicz, I. Peker, W. Tu, A.H. Barber, Stress delocalization in crack tolerant electrospun nanofiber networks, ACS Appl. Mater. Interfaces 3 (6) (2011) 1991-1996.

[27] M.F. Leong, K.S. Chian, P.S. Mhaisalkar, W.F. Ong, B.D. Ratner, Effect of electrospun poly(D,L-lactide) fibrous scaffold with nanoporous surface on attachment of porcine esophageal epithelial cells and protein adsorption, J. Biomed. Mater. Res. A 89 (4) (2009) 1040-1048. 
[28] C. Ji, N, Annabi, M. Hosseinkhani, S. Sivaloganathan, F. Dehghani, Fabrication of polyDL-lactide/polyethylene glycol scaffolds using the gas foaming technique, Acta Biomater. 8 (2) (2012) 570-578.

[29] W. Zhang, B. Chen, H. Zhao, P. Yu, D. Fu, J. Wen, X. Peng, Processing and characterization of supercritical $\mathrm{CO} 2$ batch foamed poly(lactic acid)/poly(ethylene glycol) scaffold for tissue engineering application, J. Appl. Polym. Sci. 130 (5) (2013) 3066-3073.

[30] L.M. Matuana, Solid state microcellular foamed poly(lactic acid): Morphology and property characterization, Bioresour. Technol. 99 (9) (2008) 3643-3650.

[31] Y.C. Fung, Elasticity of soft tissues in simple elongation, Am. J. Physiol. 213 (6) (1967) $1532-1544$.

[32] A.R. Cameron, J.E. Frith, J.J. Cooper-White, The influence of substrate creep on mesenchymal stem cell behaviour and phenotype, Biomaterials 32 (26) (2011) 5979-5993. 